\title{
Tail dependence for two skew slash distributions
}

\author{
Chengxiu Ling ${ }^{1 *}$ Zuoxiang Peng ${ }^{2}$ \\ ${ }^{1}$ Faculty of Business and Economics, University of Lausanne, Extranef, UNIL-Dorigny, 1015 Lausanne, Switzerland \\ ${ }^{2}$ School of Mathematics and Statistics, Southwest University, 400715 Chongqing, China
}

February 14, 2015

\begin{abstract}
Coefficient of tail dependence measures the dependencies between extreme values. In this paper, the upper tail dependence coefficients of two classes of skew slash distributions are derived. The difference of tail dependence coefficients of the two types skew slash distributions sheds light on the model choice for random variables with asymptotic dependence.

Key words and phrases: Tail dependence coefficient; Skew slash distribution; Skew-normal distribution; Variance-mean mixture.
\end{abstract}

Mathematics Subject Classification (2000) 60G15; 60G70

\section{Introduction}

Let $\boldsymbol{X}=\left(X_{1}, X_{2}\right)^{\top}$ be a bivariate random vector with marginal distribution functions (dfs) $F_{1}$ and $F_{2}$, respectively. The upper tail dependence coefficient of $\boldsymbol{X}$ is defined by

$$
\lambda_{U}=\lim _{u \uparrow 1} \mathbb{P}\left(F_{1}\left(X_{1}\right) \geq u \mid F_{2}\left(X_{2}\right) \geq u\right)
$$

provided that the limit $\lambda_{U}$ exists; see Nelsen (1999) and Embrechts et al.(2002). This quantity provides insight into the tendency for the distribution to describe joint extreme events since it measures the strength of dependence (or association) in the tails of a bivariate distribution. Generally, $\boldsymbol{X}$ is said to have asymptotic upper tail dependence if $\lambda_{U}$ is positive. In particular, trivial values $\lambda_{U}=1$ and $\lambda_{U}=0$ correspond to full dependence and independence, respectively.

\footnotetext{
${ }^{*}$ Corresponding author. Email: chengxiu.ling@unil.ch
} 
Tail independence of bivariate normal distributions was firstly addressed by Sibuya (1960) (see also Embrechts et al. (2002)) while the tail dependence of symmetry $t$-distributions was established by Demarta and McNeil (2005). Their skew-versions were further considered by Banachewicz and van der Varrt (2008) and Fung and Seneta (2010). The skew $t$-distributions are more popular and useful since it provides tail dependence of some extent as well as the skewness and heavy tails compared with (skew) normal distributions. For more related studies see, e.g., Heffernan (2000), Fung and Seneta (2011), Padoan (2011), and references therein.

In recent years, the multivariate skew-slash distributions alternatively (see (2) and (3) below for two precise definitions) have received considerable attention both in theoretical studies for their numerous stochastic properties, and in applied studies for robust statistical modeling of datasets involving distributions with skewness and heavy tails, see, e.g., conditional distributions, moments and applying skews-lash distributions to fit AIS and glass-fiber data (Wang and Genton (2006)) and characteristics functions (Kim and Genton (2011)) for skew slash distributions (3), and parameters estimation procedure such as the EM based on MLE in Arslan (2009), MLE in Lachos et al. (2010), and empirical Bayes estimations in Zareifard and Khaledi (2013) for the skew slash distributions (2). For more details see, e.g., Genç (2013) and Punathumparambatha (2013), and references therein.

Recently, tail dependence has been discussed in financial applications related to market or credit risk; see, e.g., Schmidt (2005), Durante (2013). A generalized tail dependence measure, namely tail quotient correlation coefficient was proposed by Zhang (2008) where a new test statistics of tail independence was developed; see Wu et al.(2012) for more related studies. In this paper, we shall investigate the tail dependence coefficient for two classes of skew slash distributions. The first class is defined by the normal variance-mean method. Specifically, a random vector $\boldsymbol{X}=\left(X_{1}, X_{2}\right)^{\top}$ is called to be skew slash distributed with parameters $(\lambda, \boldsymbol{\theta}, \mathbf{R})$, denoted by $\boldsymbol{X} \sim S S(\lambda, \boldsymbol{\theta}, \mathbf{R})$, if $\boldsymbol{X}$ has the following stochastic representation (see $\operatorname{Arslan}(2008,2009))$

$$
\boldsymbol{X}=\frac{\boldsymbol{\theta}}{V}+\frac{\boldsymbol{Z}}{\sqrt{V}}
$$

where $\boldsymbol{\theta}=\left(\theta_{1}, \theta_{2}\right)^{\top} \in \mathbb{R}^{2}$ and $V \sim \operatorname{Beta}(\lambda, 1), \lambda>0$ with probability density function (pdf) $f(x)=$ $\lambda x^{\lambda-1}, x \in(0,1)$, independent of $\boldsymbol{Z} \sim N_{2}(\mathbf{0}, \mathbf{R})$, a bivariate normal distribution with mean $\mathbf{0}$ and correlation matrix $\mathbf{R}$ with correlation entry $\rho \in(-1,1)$. This skew slash distribution introduces randomness into the variance and mean of a normal distribution via a beta random variable so that it is more flexible and can provide useful asymmetric and heavy-tailed extensions of their symmetric counterparts $(\boldsymbol{\theta}=\mathbf{0})$ for robust statistical modeling of datasets. For more related studies on model (2) see, e.g., generalized 
hyperbolic skew $t$-distributions in Kjersti and Ingrid (2006), skew grouped $t$-distributions in Banachewicz and van der Varrt (2008) and skew $t$-distributions in Fung and Seneta (2011).

The second class of skew slash distributions is defined as the scale-mixed skew-normal distribution (Azzalini and Dalla Valle(1996)). A random vector $\boldsymbol{X}$ is called the second type skew slash distribution, denoted by $\boldsymbol{X} \sim A S S(\lambda, \boldsymbol{\theta}, \mathbf{R})$, if $\boldsymbol{X}$ is given by

$$
\boldsymbol{X}=\frac{\mathcal{Z}}{\sqrt{V}}
$$

where $V \sim \operatorname{Beta}(\lambda, 1), \lambda>0$, independent of $\mathcal{Z}=\left(Z_{1}, Z_{2}\right)^{\top} \sim S N_{2}(\boldsymbol{\theta}, \mathbf{R})$, a bivariate skew normal distribution with pdf

$$
2 \phi_{2}(\boldsymbol{z}, \mathbf{R}) \Phi\left(\boldsymbol{\theta}^{\top} \boldsymbol{z}\right)
$$

where $\phi_{2}(\cdot, \mathbf{R})$ is the bivariate normal density function with mean $\mathbf{0}$ and correlation matrix $\mathbf{R}$, and $\Phi(\cdot)$ is the standard normal distribution function. For more related studies on model (3) see, e.g., Kim and Genton (2011), Lachos (2010) for other scaled positive variable $V$.

The goal of this paper is to establish the limit of the conditional distributions and to derive the upper tail dependence coefficient of $\boldsymbol{X}$ given by (2) and (3), respectively. Comparison with the findings of tail independence of bivariate normal (Embrechts et al.(2002)), skew-bivariate normal (Bortot (2010)); tail dependence of two skew-t distributions (Fung and Seneta(2010), Bortot (2010)), the tail dependence of the first class of skew slash distributions exist trivial values 0 or 1 for some special cases (Theorem 3.1), while the second class has wider region of tail dependence (Theorem 3.2).

The rest of the paper is organized as follows. The main results are provided in Section 3. All proofs are postponed to Section 4 .

\section{Preliminaries and notation}

In this section, we first introduce some important functions with their asymptotic properties established in Lemma 2.1 and then give Lemma 2.2 for the distribution properties of the skew slash random vector $\boldsymbol{X}$ given by (2) via the normal variance-mean mixture. 
Let $K_{\tau}(x ; \omega)$ be the incomplete modified Bessel function of the third kind with index $\tau \in \mathbb{R}$ defined by

$$
K_{\tau}(x ; \omega)=\frac{1}{2} \int_{x}^{\infty} t^{\tau-1} \exp \left(-\frac{\omega}{2}\left(t+t^{-1}\right)\right) d t, \quad x \geq 0, \omega>0 .
$$

It follows from (7.5) in Jones (2007) that for $\tau \in \mathbb{R}$

$$
K_{\tau}(0 ; \omega)=\sqrt{\frac{\pi}{2 \omega}} e^{-\omega}\left(1+\frac{4 \tau^{2}-1}{8 \omega}+o\left(\frac{1}{\omega}\right)\right), \quad \omega \rightarrow \infty
$$

Define further $P_{\tau}(a ; b)$ and $Q_{v}(x ; a)$ respectively by

$$
P_{\tau}(a ; b)=\int_{0}^{1} t^{\tau-1} \exp \left(-\frac{1}{2}\left(a^{2} t+\frac{b^{2}}{t}\right)\right) d t ; \quad Q_{v}(x ; a)=\int_{-\infty}^{x}\left(\int_{0}^{a} t^{v-1} e^{-\left(1+u^{2}\right) t} d t\right) d u, \quad x \in \mathbb{R},
$$

where $\tau>0, a, b \geq 0$ and $v \geq 1$. For simplicity, we write $\Gamma(\cdot)$ for the Euler gamma function.

The following result is about the asymptotic behaviors of $P_{\tau}(a ; b)$ and $Q_{v}(x ; a)$, respectively.

Lemma 2.1. Let $P_{\tau}(a ; b)$ and $Q_{v}(x ; a)$ be those defined as in (6). Then, we have for $P_{\tau}(a ; b)$ with $\tau>0$

$$
P_{\tau}(a ; b)= \begin{cases}\left(\frac{a^{2}}{2}\right)^{-\tau} \Gamma(\tau)(1+o(1)), & b=0, a \rightarrow \infty ; \\ \frac{b^{\tau-1 / 2}}{a^{\tau+1 / 2}} \sqrt{2 \pi} e^{-a b}\left(1+\frac{4 \tau^{2}-1}{8 a b}(1+o(1))\right), & b>0, a \rightarrow \infty ; \\ 2\left(\frac{a}{b}\right)^{-\tau} K_{\tau}(0 ; \omega)(1+o(1)), & b \rightarrow 0, a \rightarrow \infty, a b \rightarrow \omega>0\end{cases}
$$

and for $Q_{v}(x ; a)$ with $v \geq 1$ and $x \in \mathbb{R}$

$$
Q_{v}(x ; a) \rightarrow \Gamma(v) \int_{-\infty}^{x}\left(1+u^{2}\right)^{-v} d u=: Q_{v}(x ; \infty)>0, \quad a \rightarrow \infty .
$$

Recall that $\lambda_{U}$ is equivalent to

$$
\lambda_{U}=\lim _{x_{1} \rightarrow \infty} \mathbb{P}\left(X_{2} \geq F_{2}^{-1}\left(F_{1}\left(x_{1}\right)\right) \mid X_{1}=x_{1}\right)+\lim _{x_{2} \rightarrow \infty} \mathbb{P}\left(X_{1} \geq F_{1}^{-1}\left(F_{2}\left(x_{2}\right)\right) \mid X_{2}=x_{2}\right)
$$

provided that the marginal distributions are continuous (cf. Nelsen (1999), p.11, 36). In the following we derive the marginal distribution and the conditional distribution of $\boldsymbol{X}$ given by (2).

Lemma 2.2. For $\boldsymbol{X} \sim S S(\lambda, \boldsymbol{\theta}, \mathbf{R})$ given by $(2)$, let $f_{2}(\cdot)$ and $f_{1.2}\left(\cdot \mid x_{2}\right)$ denote the pdfs of $X_{2}$ and 
$X_{1.2}:=\left(X_{1} \mid X_{2}=x_{2}\right)$, respectively. Then, with $P_{\tau}(a ; b)$ given by $(6)$, we have

$$
f_{2}\left(x_{2}\right)=\frac{\lambda e^{\theta_{2} x_{2}}}{\sqrt{2 \pi}} P_{\lambda+1 / 2}\left(\left|x_{2}\right| ;\left|\theta_{2}\right|\right) ; \quad f_{1.2}\left(x_{1} \mid x_{2}\right)=\frac{e^{\beta\left(x_{1}-\rho x_{2}\right)}}{\sqrt{2 \pi\left(1-\rho^{2}\right)}} \frac{P_{\lambda+1}\left(\sqrt{x_{1}^{\prime 2}+x_{2}^{2}} ; \sqrt{\theta_{1}^{\prime 2}+\theta_{2}^{2}}\right)}{P_{\lambda+1 / 2}\left(\left|x_{2}\right| ;\left|\theta_{2}\right|\right)},
$$

where $\beta\left(1-\rho^{2}\right)=\theta_{1}-\rho \theta_{2}, x_{1}^{\prime} \sqrt{1-\rho^{2}}=x_{1}-\rho x_{2}$ and $\theta_{1}^{\prime} \sqrt{1-\rho^{2}}=\theta_{1}-\rho \theta_{2}$. Furthermore, for $\boldsymbol{\theta} \neq \mathbf{0}$

$$
\mathbb{E} e^{-s X_{1.2}}=\frac{P_{\lambda+1 / 2}\left(\left|x_{2}\right| ; \sqrt{\theta_{2}^{2}+2 \beta\left(1-\rho^{2}\right) s-\left(1-\rho^{2}\right) s^{2}}\right)}{P_{\lambda+1 / 2}\left(\left|x_{2}\right| ;\left|\theta_{2}\right|\right)} e^{-\rho x_{2} s}, \quad s \in \beta \pm \sqrt{\frac{\boldsymbol{\theta}^{\top} \mathbf{R}^{-1} \boldsymbol{\theta}}{1-\rho^{2}}} .
$$

Remark 2.1. Let $F_{2}$ be the df of $X_{2}$ for $\boldsymbol{X}=\left(X_{1}, X_{2}\right)^{\top}$ defined as in (2). Then, using (9) and Lemma 2.1, we have as $x_{2} \rightarrow \infty$ that

$$
1-F_{2}\left(x_{2}\right)= \begin{cases}\left(\theta_{2} / x_{2}\right)^{\lambda}(1+o(1)), & \theta_{2}>0 \\ \left(\widetilde{\lambda} / x_{2}\right)^{2 \lambda}(1+o(1)), & \theta_{2}=0 \\ \frac{\lambda}{2} \frac{\left|\theta_{2}\right|^{\lambda-1}}{x_{2}^{\lambda+1}} e^{-2\left|\theta_{2}\right| x_{2}}(1+o(1)), & \theta_{2}<0\end{cases}
$$

with

$$
\widetilde{\lambda}=\left(\frac{2^{\lambda-1} \Gamma(\lambda+1 / 2)}{\sqrt{\pi}}\right)^{1 /(2 \lambda)}
$$

\section{Main results}

In this section, we provide the main results on the upper tail dependence coefficient $\lambda_{U}$ of two skew slash distributions given by (2) and (3). The first result is about the upper tail dependence of the skew slash distributed random vector $\mathbf{X}$ defined by (2) via the normal variance-mean mixture model.

Theorem 3.1. Let $\boldsymbol{X} \sim S S(\lambda, \boldsymbol{\theta}, \mathbf{R})$ be defined as in (2), and let $T_{2 \lambda+1}(\cdot)$ be the student's $t$ distribution function (df) with $2 \lambda+1$ degrees of freedom. Then, with $\widetilde{\lambda}$ given by (12), we have

(1). for $\theta_{1}=\theta_{2}=0$,

$$
\lambda_{U}=2\left(1-T_{2 \lambda+1}\left(\sqrt{\frac{(2 \lambda+1)(1-\rho)}{1+\rho}}\right)\right)
$$

(2). for $\theta_{1}>0, \theta_{2}>0, \quad \lambda_{U}=1$; 
(3). for $\theta_{1}>0, \theta_{2}=0$ or $\theta_{1}=0, \theta_{2}>0$,

$$
\lambda_{U}=\int_{0}^{1}\left(1-\Phi\left(\widetilde{\lambda} u^{1 /(2 \lambda)}\right)\right) d u-\frac{1}{2 \lambda+1} \int_{0}^{1} u d\left(1-\Phi\left(\widetilde{\lambda} u^{1 /(2 \lambda)}\right)\right)
$$

(4). for the remaining cases, $\lambda_{U}=0$.

From (11) and Theorem 3.1, if both marginals posses power laws, i.e., $\theta_{1}, \theta_{2} \geq 0$, then the skew slash random vector $\boldsymbol{X}$ has asymptotic upper tail dependence. Therefore, regular varying tails play an important role in the presence of tail dependence. Theorem 3.1 shows that tail dependence of the first class of skew slash distribution exists trivial values 0 or 1 , which implies that it has extremal tail dependence (independence and full dependence), contrary to the second class of skew slash distributions showing that the tail dependence has nontrivial values.

Theorem 3.2. Let $\boldsymbol{X} \sim A S S(\lambda, \boldsymbol{\theta}, \mathbf{R})$ be defined as in (3) and let $f_{2 \lambda+1}(\cdot)$ be the probability density function (pdf) of student's $t$ distribution with $2 \lambda+1$ degrees of freedom. Then

$$
\begin{aligned}
\lambda_{U}= & \frac{\Gamma(\lambda+1 / 2)}{\Gamma(\lambda+1)}\left(\frac{1}{Q_{\lambda+1}\left(\mu_{1} ; \infty\right)} \int_{z_{0}^{\prime}}^{\infty} f_{2 \lambda+1}(z) Q_{\lambda+3 / 2}\left(\frac{\theta_{2} \sqrt{\frac{1-\rho^{2}}{2 \lambda+1}} z+\left(\theta_{1}+\rho \theta_{2}\right)}{\sqrt{1+z^{2} /(2 \lambda+1)}} ; \infty\right) d z\right. \\
& \left.+\frac{1}{Q_{\lambda+1}\left(\mu_{2} ; \infty\right)} \int_{z_{0}}^{\infty} f_{2 \lambda+1}(z) Q_{\lambda+3 / 2}\left(\frac{\theta_{1} \sqrt{\frac{1-\rho^{2}}{2 \lambda+1}} z+\left(\theta_{2}+\rho \theta_{1}\right)}{\sqrt{1+z^{2} /(2 \lambda+1)}} ; \infty\right) d z\right),
\end{aligned}
$$

where $Q_{\lambda+3 / 2}(\cdot ; \infty)$ is given by $(7)$ and

$$
\begin{aligned}
& \mu_{1}=\frac{\theta_{1}+\rho \theta_{2}}{\sqrt{1+\theta_{2}^{2}\left(1-\rho^{2}\right)}}, \quad z_{0}=\left(\left(\frac{\int_{-\infty}^{\mu_{1}}\left(1+u^{2}\right)^{-(\lambda+1)} d u}{\int_{-\infty}^{\mu_{2}\left(1+u^{2}\right)^{-(\lambda+1)} d u}}\right)^{1 /(2 \lambda)}-\rho\right) \sqrt{\frac{2 \lambda+1}{1-\rho^{2}}} ; \\
& \mu_{2}=\frac{\theta_{2}+\rho \theta_{1}}{\sqrt{1+\theta_{1}^{2}\left(1-\rho^{2}\right)}}, \quad z_{0}^{\prime}=\left(\left(\frac{\int_{-\infty}^{\mu_{2}}\left(1+u^{2}\right)^{-(\lambda+1)} d u}{\int_{-\infty}^{\mu_{1}}\left(1+u^{2}\right)^{-(\lambda+1)} d u}\right)^{1 /(2 \lambda)}-\rho\right) \sqrt{\frac{2 \lambda+1}{1-\rho^{2}}} .
\end{aligned}
$$

\section{Proofs}

Proof of Lemma 2.1 First we consider $P_{\tau}(a ; b)$. We will treat the following three cases in turn: $(1)$ $b=0, a \rightarrow \infty ;(2) b>0, a \rightarrow \infty ;(3) b \rightarrow 0, a \rightarrow \infty$ and $a b \rightarrow \omega>0$.

Case (1) as $b=0$ and $a \rightarrow \infty$. Using integration by substitution, we have

$$
P_{\tau}(a ; 0)=\left(\frac{a^{2}}{2}\right)^{-\tau} \int_{0}^{a^{2} / 2} t^{\tau-1} e^{-t} d t=\left(\frac{a^{2}}{2}\right)^{-\tau} \Gamma(\tau)(1+o(1)), \quad a \rightarrow \infty
$$


since $\int_{x}^{\infty} t^{\tau-1} e^{-t} d t=x^{\tau-1} e^{-x}(1+o(1))$ as $x \rightarrow \infty$, the claim for $P_{\tau}(a ; 0)$ follows.

Case (2) as $b>0$ and $a \rightarrow \infty$. We rewrite $P_{\tau}(a ; b)$ using $K_{\tau}(\cdot ; \cdot)$ given by (4) as

$$
P_{\tau}(a ; b)=2\left(\frac{a}{b}\right)^{-\tau}\left(K_{\tau}(0 ; a b)-K_{\tau}\left(\frac{a}{b} ; a b\right)\right) .
$$

Noting that

$$
K_{\tau}\left(\frac{a}{b} ; a b\right)=\frac{1}{2}\left(\frac{a b}{2}\right)^{-\tau} \int_{a^{2} / 2}^{\infty} t^{\tau-1} \exp \left(-\left(t+\frac{a^{2} b^{2}}{4 t}\right)\right) d t
$$

and

$$
\exp \left(-\frac{a^{2} b^{2}}{4 t}\right)=e^{-b^{2} / 2} \sum_{n=0}^{\infty}\left(\frac{u}{u+1}\right)^{n}\left(\frac{b^{2}}{2}\right)^{n}, \quad u=\frac{2 t}{a^{2}}-1
$$

we have

$$
\int_{a^{2} / 2}^{\infty} t^{\tau-1} \exp \left(-\left(t+\frac{a^{2} b^{2}}{4 t}\right)\right) d t=\left(\frac{a^{2}}{2}\right)^{\tau} \exp \left(-\frac{a^{2}+b^{2}}{2}\right) \sum_{n=0}^{\infty}\left(\frac{b^{2}}{2}\right)^{n} d_{n},
$$

with

$$
d_{n}=\frac{1}{n !} \int_{0}^{\infty} u^{n}(u+1)^{\tau-n-1} \exp \left(-\frac{a^{2}}{2} u\right) d u=: U\left(n+1 ; \tau+1 ; a^{2} / 2\right),
$$

where $U$ is the confluent hypergeometric function and $U\left(n+1 ; \tau+1 ; a^{2} / 2\right)=\left(a^{2} / 2\right)^{-n-1}(1+o(1))$ as $a \rightarrow \infty$ (cf. Chaudhry et al. (1996)). Hence,

$$
K_{\tau}\left(\frac{a}{b} ; a b\right)=\frac{a^{\tau-2}}{b^{\tau}} \exp \left(-\frac{a^{2}+b^{2}}{2}\right)(1+o(1))
$$

This together with (5) yields that

$$
\frac{K_{\tau}\left(\frac{a}{b} ; a b\right)}{K_{\tau}(0 ; a b)}=\sqrt{\frac{2}{\pi}} \frac{a^{\tau-3 / 2}}{b^{\tau-1 / 2}} \exp \left(a b-\frac{a^{2}+b^{2}}{2}\right)(1+o(1))
$$

which tends to zero as $a \rightarrow \infty$. Consequently, the claim for $P_{\tau}(a ; b)$ as $b>0, a \rightarrow \infty$ follows.

Case (3) as $b \rightarrow 0, a \rightarrow \infty$ and $a b \rightarrow w>0$. The proof is similar to that of Case (2), and thus the details are omitted here.

Next, we consider $Q_{v}(x ; a)$. Note that for all $x \in \mathbb{R}$

$$
Q_{v}(x ; a)=\Gamma(v) \int_{-\infty}^{x}\left(1+u^{2}\right)^{-v} d u-\int_{-\infty}^{x} \int_{a}^{\infty} t^{v-1} e^{-\left(1+u^{2}\right) t} d t d u .
$$


Further, recall that $v \geq 1$, and thus as $a \rightarrow \infty$

$$
0 \leq \int_{-\infty}^{x} \int_{a}^{\infty} t^{v-1} e^{-\left(1+u^{2}\right) t} d t d u \leq\left(\int_{-\infty}^{x}\left(1+u^{2}\right)^{-v} d u\right)\left(\int_{a}^{\infty} t^{v-1} e^{-t} d t\right) \rightarrow 0
$$

implying that

$$
\int_{-\infty}^{x} \int_{a}^{\infty} t^{v-1} e^{-\left(1+u^{2}\right) t} d t d u \rightarrow 0, \quad a \rightarrow \infty
$$

Therefore, for all $x \in \mathbb{R}$ and $v \geq 1$,

$$
Q_{v}(x ; a) \rightarrow \Gamma(v) \int_{-\infty}^{x}\left(1+u^{2}\right)^{-v} d u, \quad a \rightarrow \infty
$$

The proof is complete.

Proof of Lemma 2.2 Recall that $\boldsymbol{X} \mid(V=t) \sim N_{2}(\boldsymbol{\theta} / t, \mathbf{R} / t)$ with $t \in(0,1)$ given. It follows from the total probability formula that, the pdf of $\boldsymbol{X}$ defined as in $(2)$, denoted by $f_{\boldsymbol{X}}(\cdot)$, is

$$
f_{\boldsymbol{X}}(\boldsymbol{x})=\frac{\lambda e^{\boldsymbol{\theta}^{\top} \mathbf{R}^{-1} \boldsymbol{x}}}{2 \pi \sqrt{1-\rho^{2}}} \int_{0}^{1} t^{\lambda+1-1} \exp \left(-\frac{1}{2}\left(\boldsymbol{x}^{\top} \mathbf{R}^{-1} \boldsymbol{x} t+\frac{\boldsymbol{\theta}^{\top} \mathbf{R}^{-1} \boldsymbol{\theta}}{t}\right)\right) d t
$$

with $\boldsymbol{x}=\left(x_{1}, x_{2}\right)^{\top} \in \mathbb{R}^{2}$. Hence, the pdf of $X_{2}$, denoted by $f_{2}(\cdot)$, satisfies

$$
f_{2}\left(x_{2}\right)=\frac{\lambda e^{\theta_{2} x_{2}}}{\sqrt{2 \pi}} \int_{0}^{1} t^{\lambda+1 / 2-1} \exp \left(-\frac{1}{2}\left(x_{2}^{2} t+\frac{\theta_{2}^{2}}{t}\right)\right) d t
$$

Consequently, the conditional density of $X_{1.2}:=X_{1} \mid X_{2}=x_{2}$, denoted by $f_{1.2}\left(\cdot \mid x_{2}\right)$, is

$$
f_{1.2}\left(x_{1} \mid x_{2}\right)=\frac{f_{\boldsymbol{X}}(\boldsymbol{x})}{f_{2}\left(x_{2}\right)}=\frac{e^{\beta\left(x_{1}-\rho x_{2}\right)}}{\sqrt{2 \pi\left(1-\rho^{2}\right)}} \frac{P_{\lambda+1}\left(\sqrt{x_{1}^{\prime 2}+x_{2}^{2}} ; \sqrt{\theta_{1}^{\prime 2}+\theta_{2}^{2}}\right)}{P_{\lambda+1 / 2}\left(\left|x_{2}\right| ;\left|\theta_{2}\right|\right)},
$$

where $\beta\left(1-\rho^{2}\right)=\theta_{1}-\rho \theta_{2}, x_{1}^{\prime} \sqrt{1-\rho^{2}}=x_{1}-\rho x_{2}, \theta_{1}^{\prime} \sqrt{1-\rho^{2}}=\theta_{1}-\rho \theta_{2}$. Therefore, we have with $s^{\prime}=\sqrt{1-\rho^{2}} s$

$$
\mathbb{E} e^{-s X_{1.2}}=e^{-\rho x_{2} s} \mathbb{E} e^{-s^{\prime}\left(X_{1.2}-\rho x_{2}\right) / \sqrt{1-\rho^{2}}}
$$

and

$$
\mathbb{E} e^{-s^{\prime}\left(X_{1.2}-\rho x_{2}\right) / \sqrt{1-\rho^{2}}}=\frac{P_{\lambda+1 / 2}\left(\left|x_{2}\right| ;\left|\theta_{2}^{\prime}\right|\right)}{P_{\lambda+1 / 2}\left(\left|x_{2}\right| ;\left|\theta_{2}\right|\right)},
$$


with $\theta_{2}^{\prime 2}=\theta_{1}^{\prime 2}+\theta_{2}^{2}-\left(\theta_{1}^{\prime}-s^{\prime}\right)^{2}$ and $s^{\prime}$ satisfying $\theta_{1}^{\prime 2}+\theta_{2}^{2}-\left(\theta_{1}^{\prime}-s^{\prime}\right)^{2}>0$, i.e.,

$$
\theta_{2}^{\prime 2}=\theta_{2}^{2}+2 \beta\left(1-\rho^{2}\right) s-\left(1-\rho^{2}\right) s^{2}, \quad s \in \beta \pm \sqrt{\frac{\theta_{1}^{\prime 2}+\theta_{2}^{2}}{1-\rho^{2}}} .
$$

The proof is complete.

Proof of Theorem 3.1 For $\boldsymbol{\theta}=\mathbf{0}$, the skew slash random variable $\mathbf{X}$ is symmetry and has the same marginal distributions with regular varying tail index $2 \lambda$ (see (11)), and thus the claim follows by Theorem 1 (i) of Abdous (2005). Next, we derive the remaining cases, i.e., $\boldsymbol{\theta} \neq \mathbf{0}$.

To this end, we need to derive the asymptotic distribution of $W\left(x_{2}\right)$ as $x_{2} \rightarrow \infty$, where

$$
W\left(x_{2}\right):= \begin{cases}x_{2}^{-1 / 2}\left(X_{1.2}-\left(\rho x_{2}+\beta\left(1-\rho^{2}\right)\left|\theta_{2}\right|^{-1} \sqrt{x_{2}^{2}+2 \lambda}\right)\right), & \theta_{2} \neq 0 \\ x_{2}^{-2} X_{1.2}, & \theta_{2}=0 .\end{cases}
$$

For $\theta_{2} \neq 0$, it follows from Lemma 2.2 that

$$
\begin{aligned}
& \mathbb{E} e^{-s W\left(x_{2}\right)}=\exp \left(\frac{\rho x_{2}+\frac{\beta\left(1-\rho^{2}\right)}{\left|\theta_{2}\right|} \sqrt{x_{2}^{2}+2 \lambda}}{\sqrt{x_{2}}} s\right) \mathbb{E} \exp \left(-\frac{s}{\sqrt{x_{2}}} X_{1.2}\right) \\
= & \exp \left(\frac{\beta\left(1-\rho^{2}\right)}{\left|\theta_{2}\right|} \sqrt{x_{2}+\frac{2 \lambda}{x_{2}}} s\right) \frac{P_{\lambda+1 / 2}\left(\left|x_{2}\right| ; \sqrt{\theta_{2}^{2}+\frac{2 \beta\left(1-\rho^{2}\right) s}{\sqrt{x_{2}}}-\frac{\left(1-\rho^{2}\right) s^{2}}{x_{2}}}\right)}{P_{\lambda+1 / 2}\left(\left|x_{2}\right| ;\left|\theta_{2}\right|\right)}
\end{aligned}
$$

which, in view of Lemma 2.1, is asymptotically equal to

$$
\begin{aligned}
& \exp \left(\frac{\beta\left(1-\rho^{2}\right)}{\left|\theta_{2}\right|} \sqrt{x_{2}+\frac{2 \lambda}{x_{2}}} s\right)\left(\frac{\theta_{2}^{2}+\frac{2 \beta\left(1-\rho^{2}\right) s}{\sqrt{x_{2}}}-\frac{\left(1-\rho^{2}\right) s^{2}}{x_{2}}}{\theta_{2}^{2}}\right)^{\frac{\lambda}{2}} \exp \left(\left|\theta_{2}\right| x_{2}-\sqrt{\theta_{2}^{2}+\frac{2 \beta\left(1-\rho^{2}\right) s}{\sqrt{x_{2}}}-\frac{\left(1-\rho^{2}\right) s^{2}}{x_{2}}} x_{2}\right) \\
= & \exp \left(\frac{\beta\left(1-\rho^{2}\right)}{\left|\theta_{2}\right|} \sqrt{x_{2}+\frac{2 \lambda}{x_{2}}} s\right) \exp \left(\left|\theta_{2}\right| x_{2}\left[1-\left(1+\frac{2 \beta\left(1-\rho^{2}\right) s}{\theta_{2}^{2} \sqrt{x_{2}}}-\frac{\left(1-\rho^{2}\right) s^{2}}{\theta_{2}^{2} x_{2}} x_{2}\right)^{1 / 2}\right]\right)\left(1+O\left(\frac{1}{\sqrt{x_{2}}}\right)\right) \\
= & \exp \left(-\frac{\left(1-\rho^{2}\right)\left(\theta_{1}^{\prime 2}+\theta_{2}^{2}\right)}{2\left|\theta_{2}\right|^{3}} s^{2}+O\left(\frac{1}{\sqrt{x_{2}}}\right)\right)\left(1+O\left(\frac{1}{\sqrt{x_{2}}}\right)\right) \\
\rightarrow & \exp \left(-\frac{\left(1-\rho^{2}\right)\left(\theta_{1}^{\prime 2}+\theta_{2}^{2}\right)}{2\left|\theta_{2}\right|^{3}} s^{2}\right), \quad x_{2} \rightarrow \infty,
\end{aligned}
$$

where $\theta_{1}^{\prime}=\left(\theta_{1}-\rho \theta_{2}\right) / \sqrt{1-\rho^{2}}$. Therefore, by the Laplace inverse transform, we have the following convergence in distribution (denoted by $\stackrel{d}{\rightarrow}$ )

$$
W\left(x_{2}\right) \stackrel{d}{\rightarrow} Z_{1} \sim N\left(0, \frac{\left(1-\rho^{2}\right)\left(\theta_{1}^{\prime 2}+\theta_{2}^{2}\right)}{\left|\theta_{2}\right|^{3}}\right), \quad x_{2} \rightarrow \infty .
$$


For $\theta_{2}=0$, and thus $\theta_{1} \neq 0$. It follows from Lemma 2.1 and Lemma 2.2 that as $x_{2} \rightarrow \infty$

$$
\mathbb{E} e^{-s W\left(x_{2}\right)} \rightarrow \frac{2\left(\sqrt{2 \theta_{1} s}\right)^{\lambda+1 / 2} K_{\lambda+1 / 2}\left(0 ; \sqrt{2 \theta_{1} s}\right)}{2^{\lambda+1 / 2} \Gamma(\lambda+1 / 2)},
$$

which is the Laplace transform of $\theta_{1} / Y$ where $Y \sim \Gamma(1 / 2+\lambda, 1 / 2)$, a Gamma distributed random variable with shape and scale parameters $1 / 2+\lambda, 1 / 2$. Therefore

$$
W\left(x_{2}\right) \stackrel{d}{\rightarrow} \frac{\theta_{1}}{Y}, \quad x_{2} \rightarrow \infty
$$

Further, we need the asymptotic expression of the function $c\left(x_{2}\right)=F_{1}^{-1}\left(F_{2}\left(x_{2}\right)\right)$. We have by Lemma 3.1 in Banachewicz and van der Vaart (2008) that

$$
c\left(x_{2}\right)= \begin{cases}\frac{\theta_{1}}{\theta_{2}} x_{2}(1+o(1)), & \theta_{1}>0, \theta_{2}>0 \\ \frac{\theta_{1}}{\widetilde{\lambda}^{2}} x_{2}^{2}(1+o(1)), & \theta_{1}>0, \theta_{2}=0 \\ \left(\frac{2\left|\theta_{2}\right|}{\lambda+1}\right)^{1 / \lambda} \frac{\theta_{1}}{\left|\theta_{2}\right|} x_{2}^{1+1 / \lambda} \exp \left(\frac{2\left|\theta_{2}\right| x_{2}}{\lambda}\right)(1+o(1)), & \theta_{1}>0, \theta_{2}<0\end{cases}
$$

as $x_{2} \rightarrow \infty$, where $\tilde{\lambda}$ is given by (12).

Next, we give the proofs of assertions (2)-(4).

Assertion (2) as $\theta_{1}>0, \theta_{2}>0$. Using (16), (18) and $\beta\left(1-\rho^{2}\right)=\theta_{1}-\rho \theta_{2}$, we have

$$
\begin{aligned}
& \lim _{x_{2} \rightarrow \infty} \mathbb{P}\left(X_{1} \geq F_{1}^{-1}\left(F_{2}\left(x_{2}\right)\right) \mid X_{2}=x_{2}\right) \\
= & \lim _{x_{2} \rightarrow \infty} \mathbb{P}\left(W\left(x_{2}\right) \geq \frac{c\left(x_{2}\right)-\left(\rho x_{2}+\frac{\beta\left(1-\rho^{2}\right)}{\left|\theta_{2}\right|} \sqrt{x_{2}^{2}+2 \lambda}\right)}{\sqrt{x_{2}}}\right)=\mathbb{P}\left(Z_{1} \geq 0\right)=\frac{1}{2} .
\end{aligned}
$$

Similarly, $\lim _{x_{1} \rightarrow \infty} \mathbb{P}\left(X_{2} \geq F_{2}^{-1}\left(F_{1}\left(x_{1}\right)\right) \mid X_{1}=x_{1}\right)=1 / 2$. Therefore, in view of (8), we have

$$
\lambda_{U}=\lim _{x_{2} \rightarrow \infty} \mathbb{P}\left(X_{1} \geq F_{1}^{-1}\left(F_{2}\left(x_{2}\right)\right) \mid X_{2}=x_{2}\right)+\lim _{x_{1} \rightarrow \infty} \mathbb{P}\left(X_{2} \geq F_{2}^{-1}\left(F_{1}\left(x_{1}\right)\right) \mid X_{1}=x_{1}\right)=1
$$

Assertion (3) as $\theta_{1}>0, \theta_{2}=0$ and $\theta_{1}=0, \theta_{2}>0$. For this, we only present the proof of $\theta_{1}>0, \theta_{2}=0$ since another case follows by the similar arguments. Using (17) and (18), we have

$$
\lim _{x_{2} \rightarrow \infty} \mathbb{P}\left(X_{1} \geq F_{1}^{-1}\left(F_{2}\left(x_{2}\right)\right) \mid X_{2}=x_{2}\right)=\lim _{x_{2} \rightarrow \infty} \mathbb{P}\left(W\left(x_{2}\right) \geq \frac{c\left(x_{2}\right)}{x_{2}^{2}}\right)=\mathbb{P}\left(Y \leq \widetilde{\lambda}^{2}\right)
$$


where $Y \sim \Gamma(1 / 2+\lambda, 1 / 2)$ and $\widetilde{\lambda}$ is defined by (12). Similarly

$$
\begin{aligned}
& \lim _{x_{1} \rightarrow \infty} \mathbb{P}\left(X_{2} \geq F_{2}^{-1}\left(F_{1}\left(x_{1}\right)\right) \mid X_{1}=x_{1}\right) \\
= & \lim _{x_{1} \rightarrow \infty} \mathbb{P}\left(\frac{X_{2.1}-\left(\rho x_{1}+\frac{\beta^{\prime}\left(1-\rho^{2}\right)}{\left|\theta_{1}\right|} \sqrt{x_{1}^{2}+2 \lambda}\right)}{\sqrt{x_{1}}} \geq \frac{\tilde{\lambda} \sqrt{\frac{x_{1}}{\left|\theta_{1}\right|}}-\left(\rho x_{1}+\frac{\beta^{\prime}\left(1-\rho^{2}\right)}{\left|\theta_{1}\right|} \sqrt{x_{1}^{2}+2 \lambda}\right)}{\sqrt{x_{1}}}\right) \\
= & \mathbb{P}\left(Z_{1}^{\prime} \geq \frac{\tilde{\lambda}}{\sqrt{\left|\theta_{1}\right|}}\right)
\end{aligned}
$$

where

$$
\beta^{\prime}\left(1-\rho^{2}\right)=\theta_{2}-\rho \theta_{1}, \quad Z_{1}^{\prime} \sim N\left(0, \frac{\left(1-\rho^{2}\right) \boldsymbol{\theta}^{\top} \mathbf{R}^{-\mathbf{1}} \boldsymbol{\theta}}{\left|\theta_{1}\right|^{3}}\right) .
$$

Therefore, using integration by parts, we have

$$
\lambda_{U}=1-\Phi(\tilde{\lambda})+\mathbb{P}\left(Y \leq \tilde{\lambda}^{2}\right)=\int_{0}^{1}\left(1-\Phi\left(\widetilde{\lambda} u^{1 /(2 \lambda)}\right)\right) d u-\frac{1}{2 \lambda+1} \int_{0}^{1} u d\left(1-\Phi\left(\widetilde{\lambda} u^{1 /(2 \lambda)}\right)\right) .
$$

Assertion (4) as $\theta_{1} \theta_{2}<0$ and $\theta_{1}<0, \theta_{2}<0$. Here, we only present the proof of $\theta_{1}>0, \theta_{2}<0$. The other cases follow by the similar arguments and thus are omitted here. Using (16), (18) and $x_{2}^{-1} c\left(x_{2}\right) \rightarrow \infty$, we have

$$
\lim _{x_{2} \rightarrow \infty} \mathbb{P}\left(X_{1} \geq F_{1}^{-1}\left(F_{2}\left(x_{2}\right)\right) \mid X_{2}=x_{2}\right)=0, \quad \lim _{x_{1} \rightarrow \infty} \mathbb{P}\left(X_{2} \geq F_{2}^{-1}\left(F_{1}\left(x_{1}\right)\right) \mid X_{1}=x_{1}\right)=0 .
$$

Consequently, $\lambda_{U}=0$ for $\theta_{1}>0, \theta_{2}<0$. The proof is complete.

Proof of Theorem 3.2 Note that $\boldsymbol{X} \mid V=t$ is skew normal distributed with pdf $2 \phi_{2}(\boldsymbol{x} ; \mathbf{R} / t) \Phi\left(\sqrt{t} \boldsymbol{\theta}^{\top} \boldsymbol{x}\right)$ with $t \in(0,1)$ given. It follows from the total probability formula that the pdf of $\boldsymbol{X}$, denoted by $f_{\boldsymbol{X}}(\cdot)$, is

$$
\begin{aligned}
f_{\boldsymbol{X}}(\boldsymbol{x}) & =\frac{2 \lambda}{(2 \pi)^{3 / 2}|\mathbf{R}|^{1 / 2}} \int_{0}^{1} \int_{-\infty}^{\boldsymbol{\theta}^{\top} \boldsymbol{x}} t^{\lambda+3 / 2-1} \exp \left(-\frac{\boldsymbol{x}^{\top} \mathbf{R}^{-1} \boldsymbol{x}+u^{2}}{2} t\right) d u d t \\
& =\frac{2 \lambda}{(2 \pi)^{3 / 2}|\mathbf{R}|^{1 / 2}} \frac{2^{\lambda+3 / 2}}{\left(\boldsymbol{x}^{\top} \mathbf{R}^{-1} \boldsymbol{x}\right)^{\lambda+1}} \int_{-\infty}^{\frac{\boldsymbol{\theta}^{\top} \boldsymbol{x}}{\sqrt{\boldsymbol{x}^{\top} \mathbf{R}^{-1} \boldsymbol{x}}}} \int_{0}^{\frac{\boldsymbol{x}^{\top} \mathbf{R}^{-1} \boldsymbol{x}}{2}} t^{\lambda+3 / 2-1} \exp \left(-\left(1+u^{2}\right) t\right) d t d u \\
& =\frac{2 \lambda}{(2 \pi)^{3 / 2}|\mathbf{R}|^{1 / 2}} \frac{2^{\lambda+3 / 2}}{\left(\boldsymbol{x}^{\top} \mathbf{R}^{-1} \boldsymbol{x}\right)^{\lambda+1}} Q_{\lambda+\frac{3}{2}}\left(\frac{\boldsymbol{\theta}^{\top} \boldsymbol{x}}{\sqrt{\boldsymbol{x}^{\top} \mathbf{R}^{-1} \boldsymbol{x}}} ; \frac{\boldsymbol{x}^{\top} \mathbf{R}^{-\mathbf{1}} \boldsymbol{x}}{2}\right), \quad \boldsymbol{x} \neq \mathbf{0} .
\end{aligned}
$$

Consequently, the pdf of $X_{i}$, denoted by $f_{i}(\cdot)$, is given by

$$
f_{i}(x)=\frac{\lambda}{\pi} \frac{2^{\lambda+1}}{|x|^{2 \lambda+1}} Q_{\lambda+1}\left(\mu_{i} \operatorname{sign}(x) ; x^{2} / 2\right), \quad i=1,2
$$


with

$$
\mu_{1}=\frac{\theta_{1}+\rho \theta_{2}}{\sqrt{1+\theta_{2}^{2}\left(1-\rho^{2}\right)}}, \quad \mu_{2}=\frac{\theta_{2}+\rho \theta_{1}}{\sqrt{1+\theta_{1}^{2}\left(1-\rho^{2}\right)}} .
$$

Hence, we have by Lemma 2.1

$$
1-F_{2}\left(x_{2}\right)=\frac{x_{2}}{2 \lambda} f_{2}\left(x_{2}\right)(1+o(1))=\frac{\Gamma(\lambda+1)}{\pi} \frac{2^{\lambda}}{x_{2}^{2 \lambda}} \int_{-\infty}^{\mu_{2}}\left(1+u^{2}\right)^{-(\lambda+1)} d u(1+o(1))
$$

as $x_{2} \rightarrow \infty$. Consequently, as $x_{2} \rightarrow \infty$

$$
c\left(x_{2}\right)=F_{1}^{-1}\left(F_{2}\left(x_{2}\right)\right)=\left(\frac{\int_{-\infty}^{\mu_{1}}\left(1+u^{2}\right)^{-(\lambda+1)} d u}{\int_{-\infty}^{\mu_{2}}\left(1+u^{2}\right)^{-(\lambda+1)} d u}\right)^{1 /(2 \lambda)} x_{2}(1+o(1))
$$

and the pdf of $X_{1} \mid X_{2}=x_{2}$, denoted by $f_{1.2}\left(\cdot \mid x_{2}\right)$, satisfies

$$
f_{1.2}\left(x_{1} \mid x_{2}\right)=\frac{\Gamma(\lambda+1 / 2)}{\Gamma(\lambda+1)} \frac{f_{2 \lambda+1}\left(\left(x_{1}-\rho x_{2}\right) / s\left(x_{2}\right)\right)}{s\left(x_{2}\right)} \frac{Q_{\lambda+3 / 2}\left(\frac{\theta_{1} x_{1}+\theta_{2} x_{2}}{\sqrt{x_{1}^{\prime 2}+x_{2}^{2}}} ; \frac{x_{1}^{\prime 2}+x_{2}^{2}}{2}\right)}{Q_{\lambda+1}\left(\mu_{2} \operatorname{sign}\left(x_{2}\right) ; \frac{x_{2}^{2}}{2}\right)}
$$

where $f_{2 \lambda+1}(\cdot)$ is the pdf of student's $t$ with $2 \lambda+1$ degrees of freedom and

$$
x_{1}^{\prime} \sqrt{1-\rho^{2}}=x_{1}-\rho x_{2}, \quad s\left(x_{2}\right)=\sqrt{\frac{\left(1-\rho^{2}\right) x_{2}^{2}}{2 \lambda+1}} .
$$

Hence, we have by the dominated convergence theorem and Lemma 2.1 that

$$
\begin{aligned}
& \lim _{x_{2} \rightarrow \infty} \mathbb{P}\left(X_{1} \geq F_{1}^{-1}\left(F_{2}\left(x_{2}\right)\right) \mid X_{2}=x_{2}\right) \\
= & \frac{\Gamma(\lambda+1 / 2)}{\Gamma(\lambda+1)} \frac{1}{Q_{\lambda+1}\left(\mu_{2} ; \infty\right)} \int_{z_{0}}^{\infty} f_{2 \lambda+1}(z) Q_{\lambda+3 / 2}\left(\frac{\theta_{1} \sqrt{\frac{1-\rho^{2}}{2 \lambda+1}} z+\left(\theta_{2}+\rho \theta_{1}\right)}{\sqrt{1+z^{2} /(2 \lambda+1)}} ; \infty\right) d z,
\end{aligned}
$$

where

$$
z_{0}=\lim _{x_{2} \rightarrow \infty} \frac{c\left(x_{2}\right)-\rho x_{2}}{s\left(x_{2}\right)}=\left(\left(\frac{\int_{-\infty}^{\mu_{1}}\left(1+u^{2}\right)^{-(\lambda+1)} d u}{\int_{-\infty}^{\mu_{2}}\left(1+u^{2}\right)^{-(\lambda+1)} d u}\right)^{1 /(2 \lambda)}-\rho\right) \sqrt{\frac{2 \lambda+1}{1-\rho^{2}}}
$$

Similarly

$$
\begin{aligned}
& \lim _{x_{1} \rightarrow \infty} \mathbb{P}\left(X_{2} \geq F_{2}^{-1}\left(F_{1}\left(x_{1}\right)\right) \mid X_{1}=x_{1}\right) \\
= & \frac{\Gamma(\lambda+1 / 2)}{\Gamma(\lambda+1)} \frac{1}{Q_{\lambda+1}\left(\mu_{1} ; \infty\right)} \int_{z_{0}^{\prime}}^{\infty} f_{2 \lambda+1}(z) Q_{\lambda+3 / 2}\left(\frac{\theta_{2} \sqrt{\frac{1-\rho^{2}}{2 \lambda+1}} z+\left(\theta_{1}+\rho \theta_{2}\right)}{\sqrt{1+z^{2} /(2 \lambda+1)}} ; \infty\right) d z,
\end{aligned}
$$


with

$$
z_{0}^{\prime}=\left(\left(\frac{\int_{-\infty}^{\mu_{2}}\left(1+u^{2}\right)^{-(\lambda+1)} d u}{\int_{-\infty}^{\mu_{1}}\left(1+u^{2}\right)^{-(\lambda+1)} d u}\right)^{1 /(2 \lambda)}-\rho\right) \sqrt{\frac{2 \lambda+1}{1-\rho^{2}}} .
$$

The desired result follows by (23) and (24).

Acknowledgement: The authors are grateful to the Editor-in-Chief (Professor. Dr. Yazhen, Wang) and the referees for their careful reading and helpful suggestions which greatly improved the paper. C. Ling was supported by the Swiss National Science Foundation Grant 200021-1401633/1 and the project RARE -318984; Z. Peng was supported by the National Natural Science Foundation of China grant no.11171275 and the Natural Science Foundation Project of CQ no. cstc2012jjA00029.

\section{References}

[1] Abdous, B., Anne-Laure Fougeres, A.L. And Ghoudi, K., 2005. Extreme behaviour for bivariate elliptical distributions. The Canadian Journal of Statistics. 33, 317-334.

[2] Arellano-Valle, R.B., Genton, M.G. and Loschi, R.H., 2009. Shape mixtures of multivariate skewnormal distributions. Journal of Multivariate Analysis. 100, 91-101.

[3] Arslan, O., 2008. An alternative multivariate skew-slash distribution. Statistics and Probability Letters. 78, 2756-2761.

[4] Arslan, O., 2009. Maximum likelihood parameter estimation for the multivariate skew-slash distribution. Statistics and Probability Letters. 79, 2158-2165.

[5] Azzalini, A. and Dalla Valle, A., 1996. The multivariate skew-normal distribution. Biometrika. 83, $715-726$.

[6] Banachewicz, K., and van der Vaart, A., 2008. Tail dependence of skewed grouped t-distributions. Statistics and Probability Letters. 78, 2388-2399.

[7] Bortot, P., 2010. Tail dependence in bivariate skew-normal and skew- $t$ distributions. (http://www2.stat.unibo.it/bortot/ricerca/paper-sn-2.pdf).

[8] Cabral, C.R.B., Lachos, V.H. and Prates, M.O., 2012. Multivariate mixture modeling using skewnormal independent distributions. Computational Statistics and Data Analysis. 56, 126-142. 
[9] Chaudhry, M.A., Temme, N.M. and Veling, E.J.M., 1996. Asymptotics and closed form of a generalized incomplete gamma function. Journal of Computational and Applied Mathematics. 67, 371-379.

[10] Demarta, S. and McNeil, A.J., 2005. The $t$ copula and related copulas. International Statistical Review. 73, 111-129.

[11] Durante, F., 2013. Copulas, tail dependence and applications to the analysis of financial time series. In Bustince, H., Fernandez, J., Mesiar, R. and Calvo, T. (Eds.), Aggregation functions in Theory and in Practice. vol. 228. 17-22. Springer, New York.

[12] Embrechts, P., McNeil, A., and Straumann, D., 2002. Correlation and dependence in risk management: properties and pitfalls. In Dempster, M.H.A.( editor), Risk management: Value at Risk and Beyond, pages 176-233. Cambridge University Press, Cambridge.

[13] Fung, T. and Seneta, E., 2010. Tail dependence for two skew $t$ distributions. Statistics and Probability Letters. 80, 784-791.

[14] Fung, T. and Seneta, E., 2011. Tail dependence and skew distributions. Quantitative Finance. 11, $327-333$.

[15] Genç, A.İ., 2013. A skew extension of the slash distribution via beta-normal distribution. Stat Papers. 54, 427-442.

[16] Heffernan, J.E., 2000. A directory of coefficients of tail dependence. Extremes. 3, 279-390.

[17] Jones, D.S., 2007. Incomplete Bessel function I. Proceedings of the Edinburgh Mathematical Society. 50, 173-183.

[18] Kim, H.M., Genton, M.G., 2011. Characteristic functions of scale mixtures of multivariate skewnormal distributions. Journal of Multivariate Analysis. 102, 1105-1117.

[19] Kjersti A. and Ingrid H.H., 2006. The generalized hyperbolic skew Student's t-distribution. Journal of Financial Econometrics. 4, 275-309.

[20] Lachos, V.H., Ghosh, P., and Arellano-Valle, R.B., 2010. Likelihood based inference for skew-normal independent linear mixed models. Statistics Sinica. 20, 303-322.

[21] Nelsen, R.B., 1999. An Introduction to Copulas. Lecture Notes in Statistics. 139, Springer Verlag.

[22] Padoan, S.A., 2011. Multivariate extreme models based on underlying skew $t$ and skew-normal distributions. Journal of Multivariate Analysis. 102, 977-991. 
[23] Punathumparambatha, B., 2013. A new family of skewed slash distributions generated by the Cauchy kernel. Communications in Statistics-Theory and Methods. 42, 2351-2361.

[24] Schmidt, R., (2005). Tail dependence. In Čížek, P., Hädle, W. and Weron, R. (Eds.), Statistical Tools for Finance and Insurance, pages 65-91. Springer, New York.

[25] Sibuya, M., 1960. Bivariate extreme statistics, I. Ann. Inst. Statist. Math. 11, 195-210.

[26] Wang, J. and Genton, M., 2006. The multivariate skew-slash distribution. Journal of Statistal Planning Inference. 136, 209-220.

[27] Wu, J., Zhang, Z. and Zhao, Y., 2012. Study of the tail dependence structure in global financial markets using extreme value theory. Journal of Reviews on Global Economics. 1, 62-81.

[28] Zareifard, H. and Khaledi, M.J., 2013. Empirical Bayes estimation in regression models with generalized skew-slash errors. Communications in Statistics - Theory and Methods. 42, 1105-1123.

[29] Zhang, Z., 2008. Quotient correlation: a sample based alternative to Pearson's correlation. Annals of Statistics. 36, 1007-1030. 